\title{
Effect of Coupling Chain Length on the Electro-Optic Properties of Side Chain Liquid Crystal Polymers Adding $\mathrm{BaTiO}_{3}$ Nanoparticles
}

\author{
Zahraa kadhum ${ }^{\dagger}$, Alaa shaker ${ }^{*}$ and Falih Hussien ${ }^{\dagger}$ \\ $\dagger$ Power Mechanics, AL-Furat Al-Awsat Technical University, Babylon Iraq \\ * Power Mechanics, AL-Furat Al-Awsat Technical University, Babylon Iraq \\ ${ }^{\dagger}$ Collage of Engineering, Thi- QarUniversity, Thi-Qar Iraq
}

\begin{abstract}
A series of siloxine centered side chain liquid crystal polymers have been arranged with asystematic variation in spacer length. All the polymer shown a nematic phase, for which the nematic-isotropic transition temperature increased as the spacer length bigger. The mesogenic group has syano head groups $(\mathrm{C} \boldsymbol{\Xi})$ ) to which positive dielectric anisotropic ( $\boldsymbol{\varepsilon} \boldsymbol{\Delta}>$ $0)$ is accredited to bind the mesgneic group with the backbone we have used elastic spascer of $\left(\mathrm{CH}_{3}\right)$ which changes from (2) to (6) units. The nematic cells that have been arranged have planer reorientation to notice the changes inside electro-optic cell Frederick's transition manner. It found that with increasing spacer length (n) of polymer the threshold voltage is let down. It been also institute that there is a reverse relationship between temperature and response times this scholarship also deals with the effect of elastic spacer on response times and it has been learned the response times of the polymer with short spacers are longer than response times of the polymers with long spacers, in addition the response times of the three polymers are in reorientation the spacer length lastly we have been found that there the increase in applied voltages lead to the lessening in turn on times ( $\left.\boldsymbol{\tau}_{\mathrm{on}}\right)$ with the turn off times $\left(\boldsymbol{\tau}_{\mathrm{off}}\right)$. The difference of the threshold voltage ascends from changes to the intrinsic curvature elasticity rather than to the differences in the orientation demand. Keywords: Barium Titanate nanoparticle's, Polysiloxane , electro - optic properties.
\end{abstract}

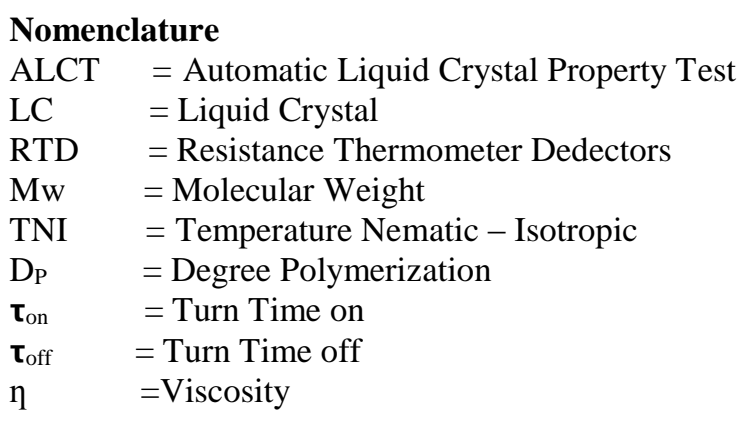

\section{Introduction}

The electro-optic Liquid crystal property is the description of the intermediate situation that is limited configured in the range between solid-state, where the movement of molecules bound with high system (orientation) and between positions isotropic liquid in which the molecules owns freedom of movement (no orientation), and it is found between them by thermal or cold action or by the effect of a solvent. They are sticky, jelly-like materials that resemble liquids in certain sides. Liquid crystals must be geometrically vastly anisotropic (having unlike optical properties in different directions) [1]. The countless class of organic molecules that does not display a single transition from solid to liquid but rather a chains (more than one) of transition between the solid (anisotropic) to the ordinary (isotropic) liquid as their temperature is raised. These newfangled phases have optical and structural possessions between the crystalline solid and the isotropic liquid. For this cause, these phases are revealed to liquid crystalline phase, and the materials which from them a change in phase are often mentioned to thermo tropic liquid crystal and a more proper name is transitional phases, where there are two distinct types of behavior in liquid crystals, lyotropic and thermotropic liquid crystal [2].In order to distinguish the nature of liquid crystals, one should recall the structure crystal, here the middles of masses related long range order, subsequently the $\mathrm{x}$-ray diffraction shapes are showing sharp Bragg reflection. Because of their vastly ordered and monotonous structure, crystals give diffraction patterns of sharp Bragg reflection, and are perfect for analyzing the structure of solids[3,4]. The crystal have long range directive in three dimensions as well as to 
orientational order while in the liquid crystalline phase the at slightest one of these dimensions is removed with present short orientational order between the molecules. Furthermore, isotropic liquids are contained on short range order between the cores of masses. The $\mathrm{x}$ - ray diffraction patterns now only show broad, diffuse reflection.There are two fundamental orderings in the materials: positional order and orientational order, at low temperatures, matter exhibits a crystal state. The atoms or molecules are arranged regularly and periodically. Both position order and orientation order exhibit a long range order[5,6]. When heating, the matter may become an isotropic liquid through two ways, by losing the orientational order first while retaining the translational order, and hence becoming a plastic crystal, or alternatively, by losing the translational order first while keeping the orientational order. The basic condition for the liquid crystal is possess a form molecularly anisotropic significantly $[\mathbf{7 , 8}]$.

\section{Experimental}

The procedures followed for the construction of electro optic cells with predefind director orientation in these investigations were similar to those developed for low molecular weight liquid crystal.[Blazers Z20 tin oxide] coated glass slides were used for all electro - optic cells constructed in this work. This was easily cut and was sufficiently flat to allow the fabrication of cells with parallel plate separation to within a few seconds of arc . The glass area was cut into plate of approximately $6 \mathrm{~cm}^{2}$, and etched using HCL acid with zinc metal powder as a catalyst to give a $2 \mathrm{~cm}^{2}$ electrode surface. Each glass plate was then washed hand- by - hand in soup and water and cleand in an ultrasonic bath for 30 minutes at $65 \mathrm{C}^{\circ}$. The plates were then rinsed in distilled water and dried in an oven for 20 minutes at $60 \mathrm{C}^{\circ}$. This treatment produced plates sufficiently clean for the surface treatment procedure, which is the subject of the next section. In this work one technique was used to achieve uniform planer alignment of the liquid crystal director. The cell electrodes were coated with a thin layer of polyimide precursor [consisting of a 5\% solution of Rodehftal 322 (Rh one Poulenc chemical Ltd .) in dimethyl formaimed ] , using a spin - coater running at 4,500 rpm, these coated slides were heated in an oven for 30 minutes at $80 \mathrm{C}^{\circ}$, they were then rubbed at room temperature in a single direction with a cloth using controlled repeatable procedure, heated again for 30 minutes at $130 \mathrm{C}^{\circ}$, finally it will be putted for one hour at $200 \mathrm{C}^{\circ}$ in the oven.

\subsection{Experimental Set - up :}

The most important three devices were used in this work for electro - optic measurement are :

1.MK1000

2.HCS402

3.ALCT

\subsubsection{The MK1000}

series temperature controller offers precision, accuracy, and stability for temperature measurement and control. It provides temperature resolution and precision to $0.001 \mathrm{C}^{\circ}$, accommodates both Pltatinum RTD and individually calibrated thermistors as temperature sen, and plates, or chucks. Two operation modes, keypad operation using the front panal of the controller, or software control though pc as well as, adjustable ramp ( rate of heating / cooling) to user set temperature point. Programmable operation command set. Precisely controls temperature to $0.001 \mathrm{C}^{\circ}$ option save temperature data to the computer . RTD thermositor or thermocouple, LC cell holders for many types of LC cells.

\subsubsection{Automatic liquid crystal property tester -ALCT}

The automatic liquid crystal measurement systems (ALCT) are USB based instruments which can be connected conveniently to both desktop and laptop computers (Instec Company). Depending on options, the ALCT's easy-to-use software allows for the measurement of material parameters for both positive and negative dielectric liquid crystals, and material parameters of liquid crystal such as dielectric constants $\varepsilon_{\perp}$ and $\varepsilon_{\mid}$,elastic constants splay and bend $\left(\mathrm{K}_{11}\right.$ and $\left.\mathrm{K}_{33}\right)$, voltage holding ratio measurement system, optical switching time, optical transmittance vs voltage-threshold voltage $\mathrm{U}_{c}$, polarization current $\mathrm{I}_{\mathrm{p}}$ and Viscosity, the ALCT can also be integrated with instec's microscope hot and cold stages and temperature controllers, enabling the user to analyze the temperature dependence of the above parameters.

\subsubsection{Microscope hot /cold Stage - HCS302}

HCS302 has the following features (Instec Company):

1. Dual-heater microscope hot and cold stage for increased temperature gradient uniformity, this is demonstrated in Fig. (1).

2. Programmable precision temperature control from $\left(200-600^{\circ} \mathrm{C}\right)$.

3. Controlled fast heating and cooling rate.

4. Large viewing aperture.

5. Variable sample chamber height.

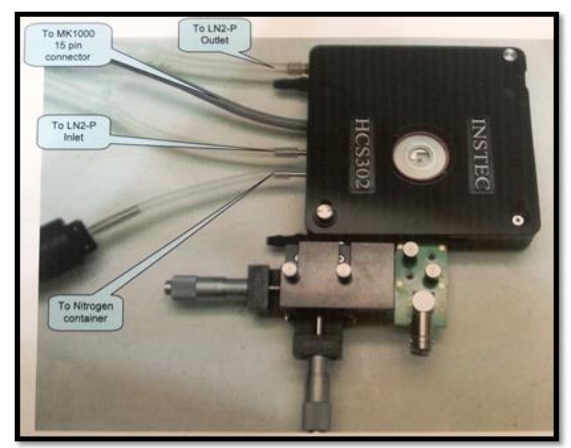

\section{Results}

Fig.1 Microscope hot /cold Stage - HCS302

This study is concerned with evaluating the static electro-optic properties for the range of liquid crystal polymers shown in Table (1) as a function of temperature. 
By using the experimental arrangement described in the previous section, it is possible to determine the threshold electric field required to distort the surface realigned director orientation . Figures. (4-8)show the variation of the transmitted light intensity as a function of the applied voltage and the required voltage for each material in this chapter to complete switching. For all polymers, the dielectric anisotropy is positive. The electric field deforms an initial planar surface voltages . For all alignment and in this case of the geometry is dominated by the splay component of the curvature elasticity constant $\mathrm{K}_{11}$ for small deformations [9]. The polymer system has a number of particular problems which in turn play significant role in the evaluation of $\mathrm{V}^{\text {th }}$ for thepo lymer with high viscosity . Also, the variance in the length of the spacer in this series of polysiloxane based side chain liquid crystal polymers affect onmthe phase behavior, the order parameter and electroptic properties. By studying response times for the five polymers, they can be divided intopolymers which have relatively long side chains (P.1, P. 2, P.3) and polymers which have relatively short side chains (P.4, P.5). It reasonable to assume that as the coupling chain shortened, so the coupling between the mesogenic unit and the polymer chain is increase. If ordering increases as a result of this coupling, it is natural to expect the transitions to rise. In practice, as shown in Table (1), the opposite is observed. This suggests that the emphasis on the role of the polymer chain should be one of in case of polymer 4 , polymer 5 ordering through coupling. Clearly there is a subtle balance between these possibilities and small variation can lead to marked effects. the variation of the nematic -isotropic variation transition temperature with spacer length, as shown in Fig (9) . This figure appears to have some element of the well-known odd-even effect observed in low molar mass liquid crystalline materials and in main -chain liquid crystal polymers . The increase in the glass transition temperature with decreasing spacer length follows expectations based on the restricted motions of the polymer backbone segments. Clear trends are observed in the threshold voltage variation as a function of both temperature and spacer length.as shown in Figures (10-12) it emphasized that considerable care and effort has been taken to exclude from these experiments the time dependent effects of the high viscosities associated with polymeric liquid crystal systems. In part, the increase of the threshold voltage with decreasing temperature is expected on the basis of an increasing order parameter. The origin of the variation of the threshold voltage with spacer length is not so clear. If we make any correction for the slight increase in the dielectric anisotropy with decreasing spacer length, then this will simply enhance the rapidly increasing threshold voltage at low values of $\mathrm{n}$. that this rapidly rising threshold voltage with changing coupling chain length arises in part from factors beyond those related to the varying order parameter. In the spirit of the approach of Nehring and Saupe [10], we may relate this to some increase in the intrinsic curvature elasticity of the system.
Table 1 Molecular weight and phase transition data polysiloxane.

\begin{tabular}{|c|c|c|c|c|c|}
\hline $\mathrm{TNI}^{\circ} \mathrm{C}$ & $\mathrm{T}_{\mathrm{g}}{ }^{\circ} \mathrm{C}$ & $\mathrm{DP}_{\mathrm{P}}$ & $\mathrm{M}_{\mathrm{w}}$ & $\begin{array}{l}\mathrm{N} \\
\text { spacer } \\
\text { length }\end{array}$ & Polymer \\
\hline$\pi$ Tr. & $\sum \Lambda$ & Tr. & $1.7 \times 10^{6}$ & 6 & 1 \\
\hline Iro & $\varepsilon$. & $1 . r$ & $8.1 \times 10^{5}$ & 0 & r \\
\hline $1 \cdot \varepsilon$ & r & 10 & $7.2 \times 10^{4}$ & $\varepsilon$ & $r$ \\
\hline VV & 17 & V & $4.5 \times 10^{3}$ & $r$ & $\varepsilon$ \\
\hline 71 & $\varepsilon$ & $\varepsilon$ & $3.1 \times 10^{3}$ & $r$ & 0 \\
\hline
\end{tabular}

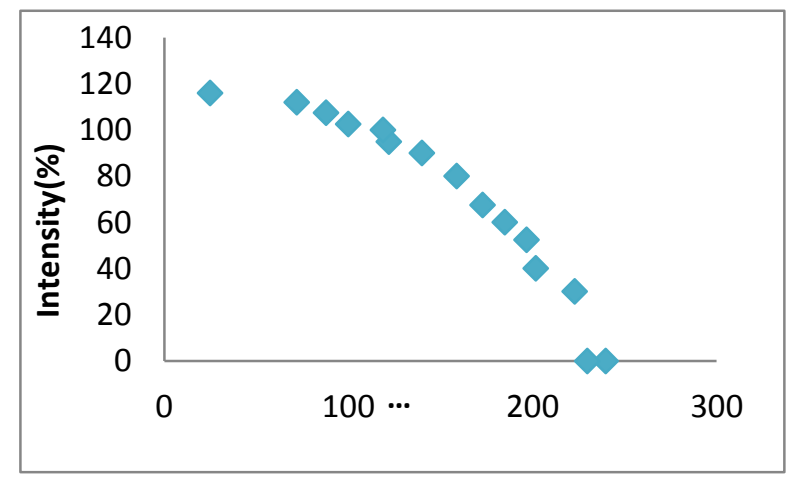

Fig. 2 The relation between voltage and intensityof (p1).

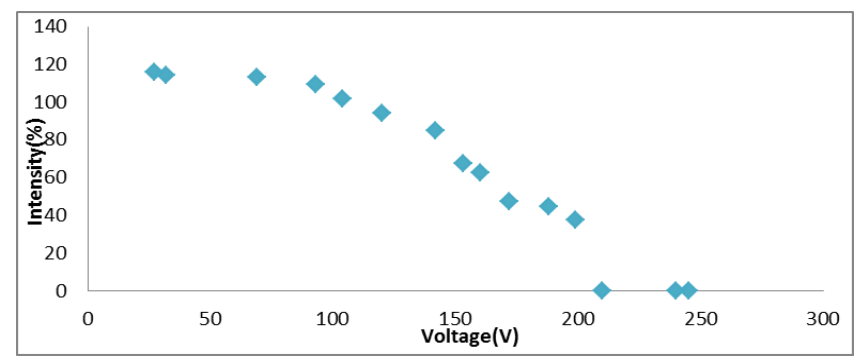

Fig. 3The relation between voltage and intensity of (p2).

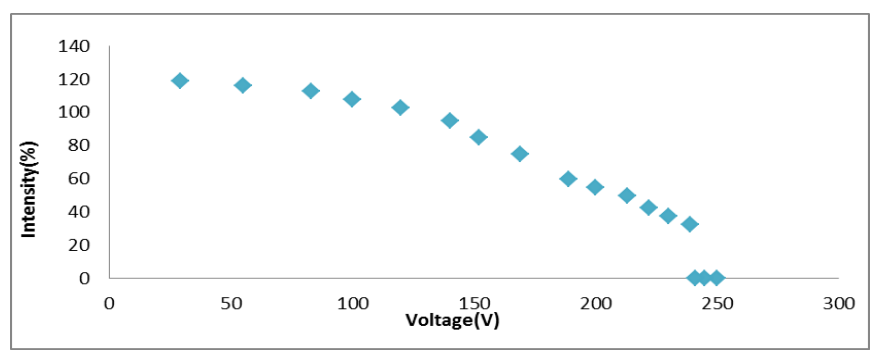

Fig.4The relation between voltage and intensity of (p3). 


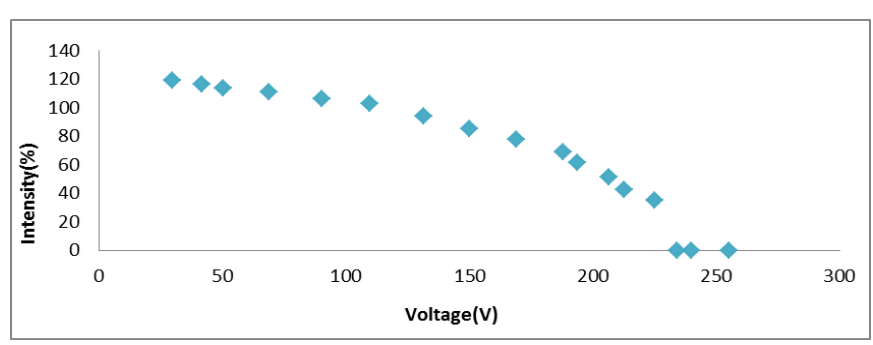

Fig.5The relation between voltage and intensity of(p4).

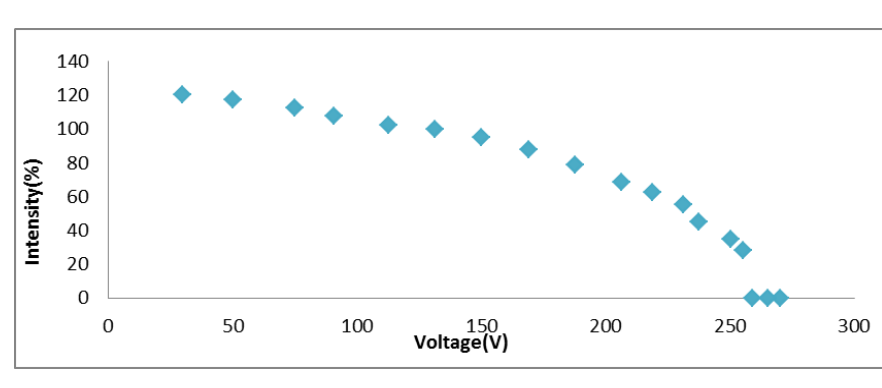

Fig.6 The relation between voltage and intensity of (P5).

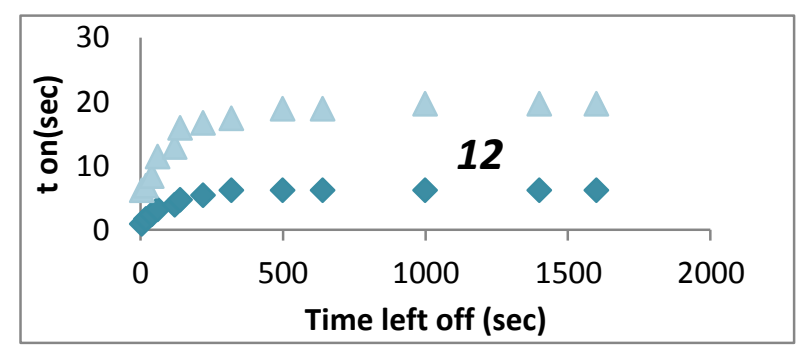

Fig. $7 \tau_{\text {on }}$ variation as a function of $\tau_{\text {off }}$ at different temperature p.5

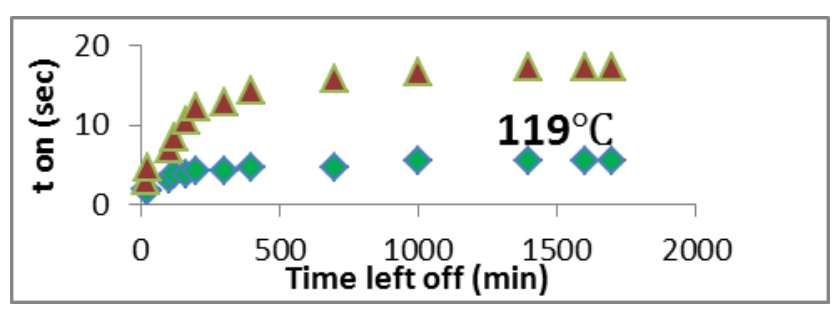

Fig.8 $\tau_{\text {on }}$ variation as a function of $\tau_{\text {off }}$ at different temperature $\mathrm{p} 4$.

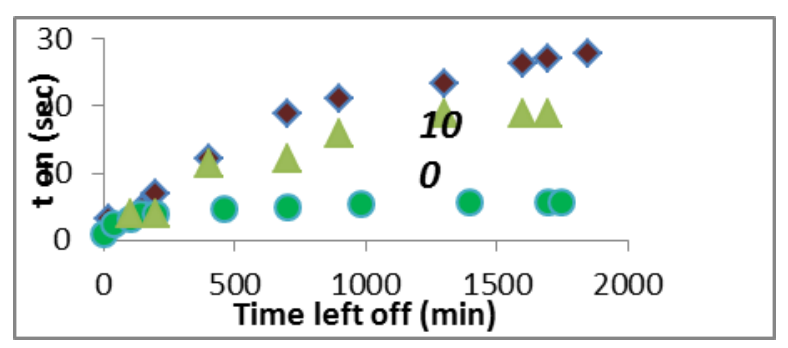

Fig.9 $\tau_{\text {on }}$ variation as a function of $\tau_{\text {off }}$ at different temperature p3.

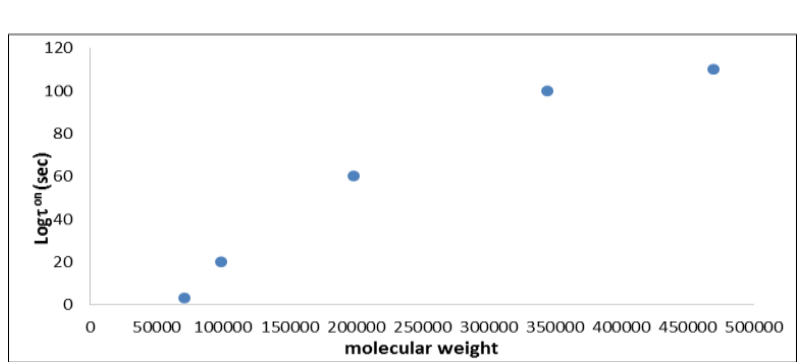

Fig.10 Corrected $\tau^{\text {on }}$ as a function of molecular weight

\section{Conclusions}

The study concludes the following:

1 .This study has focused on the properties of a series of siloxan based liquid crystal polymers with identical mesogenic units and adding barium titanate but with differing lengths of the coupling chain .

2. Distinct and significant trends are observed in both the phase transition behavior and in the static electro -optic properties these rend support the concept that an increasing spacer chain length decouples to a certain extent the motions of the mesogenic side-groups from those of the polymer backbone.

$r$. The variations in the threshold voltage with changing spacer length arise from factors over and above the minor variations observed in the orientational order. These additional factors are related to the constraints on the alignment of the mesogenic units arising from the relatively close coupling to the polymer chain .

$\varepsilon$. The relationship between $\left(\tau_{\text {on }}\right)$ and the space length is a positive relationship and this is what achieves relationship Fox $: \tau_{\text {on }} \propto \eta \propto \mathrm{Mw},\left(\tau_{\text {on }}\right.$ turn on times, $\eta$ viscosity, Mw molecular weight ).

5. It tried to prove that $\tau_{\text {on }}$ a function to $\mathrm{T}$ in other words, $\tau_{\text {on }} \propto \eta \mathrm{T}$ that we got a good result. 
7 . Through our study, it is found that the phase behavior of polymers $(6,5,4)$ shows nematic and smectic phases either of polymers $(3,2)$ we observed only nematic phase.

7. The addition of $\mathrm{BaTiO}_{3}$ nanoparticles led to an improvement in optical properties through a decrease in response time with an increase in the length of the spacer.

\section{References}

[1] Brown, G.H, and wolken. J.J., "liquid crystals and biological structures." ISBN: 0121368505, $3^{\text {rd }}$ Edition, Academic press, London, (1979).

[2] Kumar, S., "Liquid crystals: Experimental study of physical properties and phase transition" Cambridge University press, UK, (2001).

[3] Cowley, J.M., "Diffraction physics," Elsevier Silence Publishers, North-Holland, Amsterdam, (1995).

[4] Snigirev.A., Bjeoumikhov, A., Erko, A., Snigireva, I., Grigoriev, M., Yunkin, V., Erko, M., and Bjeoumikhova, S., "Two-step hard X-ray focusing combining Fresnel zone plate and single-bounce ellipsoidal capillary," J. Synchrotron Rad, Doi: 10.1107/S0909049507025174 (2007).

[5] Barim.G, "Lyotropic Liquid Crystalline Mesophases of Lithium Salt - Nonioic Surfactants." Proceedings of International Conference on Organized Molecular Films (Icomf14) - Lb14, Paris, (France), Jul 10-Jul 13 (2012).

[6] Lehmann, $M$ and Seltmann, J., "Low temperature enantiotropic nematic phases from V- shaped, shapepersistent molecules," Beilstein J. Org. Chem., 5, No. 73 (2009).

[7] Vertogen, G. and De Jeu, Wim H. "Thermotropic liquid crystals: zfundamentales, " Springer-Verlag Berlin Heidelberg, (1988).

[8] Chandrasekhar, S. Sadashiva, B. K., and Suresh, K. A., "Liquid crystals of disc - Like molecules," Pramana, India, Vol. 9, PP. 471-480, (1977).

[9] Finkel Mann. H, Kie Chle.U. And Rehage.G, Mol. Cryst. Liq . Cryst, 4,9,92,(1983)

[10] Nehring . J. and Saupe. A, J. Chem. Phys,5, 23,123,(2006). 